ESJ Humanities

\title{
Règlement de litiges fonciers et rhétorique discursive en pays atchan (en Ébrié en Côte d'Ivoire)
}

\author{
Happy Rosalie Lolo Monney \\ Léon Yepri \\ École Normale Supérieure(ENS) d'Abidjan, Côte d'Ivoire
}

Doi:10.19044/esj.2022.v18n2p67

Submitted: 03 January 2022

Accepted: 29 January 2022

Published: 31 January 2022
Copyright 2022 Author(s)

Under Creative Commons BY-NC-ND

4.0 OPEN ACCESS

Cite As:

Lolo Monney H.R. \& Yepri L. (2022). Règlement de litiges fonciers et rhétorique discursive en pays atchan (en Ébrié en Côte d'Ivoire). European Scientific Journal, ESJ, 18 (2), 67. https://doi.org/10.19044/esj.2022.v18n2p67

\section{Résumé}

Cet article vise à révéler les grandes composantes de la rhétorique discursive lors du règlement de litiges fonciers en pays atchan (peuple ébrié situé au sud-est de la Côte d'Ivoire). Le jeu des échanges verbaux autour des lois coutumières sont le reflet d'une vision du monde qui met le bien-être communautaire au centre de toute préoccupation. Nous avons utilisé comme technique de recherche qualitative l'observation d'un fait socioculturel. Aussi, avons -nous assisté à une assemblée de notables, pour régler un litige foncier dans le village d'Akoue-djeme. Le déroulé de cette procédure judiciaire coutumière a été transcrit, puis analysé selon l'organisation de la rhétorique discursive. Ce travail a permis de découvrir les trois grandes parties de la séance du règlement dudit conflit. La rhétorique du discours social atchan est faite de jeux intertextuels ponctués par des formules ritualisées et proverbiales.

Mots clés : Rhétorique discursive, litige foncier, discours social, échange verbal, grammaticalité culturelle 


\title{
Land Dispute Resolution and Discursive Rhetoric
}

\author{
Happy Rosalie Lolo Monney \\ Léon Yepri \\ École Normale Supérieure(ENS) d'Abidjan, Côte d'Ivoire
}

\begin{abstract}
This article aims at showing the major components of the discursive rhetoric during land dispute resolution among the Atchan community (The Ebrie people located in the South west of Cote d'Ivoire). The way of going about the oral communication talking about customary laws truly reflects a world vision that sets the community well-being as the prior preoccupation. The qualitative methodology is the one used. We took part in a gathering of dignitaries solving a land dispute in the Akoue-djeme village. The event of this customary court has been transcribed and analyzed according to the discursive rhetoric organization. This study enabled us to discover the three major components of the sitting. The rhetoric of the Atchan social speech is made up with inter-textual games with rituals and proverbs.
\end{abstract}

Keywords: Discursive rhetoric, land dispute, social speech, oral communication, grammatical culture

\section{Introduction}

Que la parole traditionnelle, notamment africaine, soit un art doublé d'un rituel, plus personne ne le conteste aujourd'hui ; surtout lorsque celle-ci est produite dans des circonstances solennelles, voire exceptionnelles. «Le fait de n'avoir pas d'écriture ne prive pas pour autant l'Afrique d'avoir un passé, et une connaissance. », (HAMPATÉ BA, A., 1972 :22).

Dans les sociétés africaines, en effet, tout particulièrement en pays atchan, la parole est définie comme une pratique relevant d'un code marqué par des exigences conventionnelles, sous l'aspect de règles et de procédures. Les contraintes s'y attachent et il est difficile d'y échapper, sinon l'on se verrait rappeler à l'ordre, parfois même au moyen d'une « amende », en nature ou en argent ou d'une sanction disciplinaire sociale...Cela est observé dans certaines situations telles les assemblées rituelles, lors des fêtes de classes d'âges, de génération, et de différents ou litiges...

Une telle situation requiert, pour l'utilisation de la parole, un art d'une grande habileté, d'une extrême efficacité ; toutes choses qui donnent à la parole son caractère sacré puisqu'elle est la croyance, elle remonte aux Ancêtres, ceux-ci sont proches de l'être suprême. 
L'exposé qui va suivre tentera, en guise d'illustration de montrer les différentes grandes étapes de la réalisation de la parole en pays atchan, lors d'un règlement de litige foncier entre les membres d'une même famille. Il s'agira de mettre également en relief, la rhétorique du discours social, enrichi de composantes et de spécificités culturelles du milieu de référence. Enfin, nous allons déterminer, l'impact de la production de ladite parole dans son contexte, ce, en termes d'effets illocutoires et perlocutoires, au regard de l'analyse du discours. Magie et force, la parole exerce, d'une manière ou d'une autre un attrait certain sur les destinataires.

Ce sera somme toute l'occasion de montrer, par un procédé d'insistance, l'actualité et l'intérêt plus que certain de l'oralité, art de la parole et somme de connaissances hérités des ancêtres.

\section{Une brève présentation de la culture atchan}

L'appellation atchan relève de la tradition de l'espace socioculturel et géographique des tchaman ${ }^{1}$.

«Le terme atchan désigne indivisément le pays, la société, la culture et l'ethnie des tchaman. »,(DJOKE, B., 2014 :36). Les tchaman venus du Ghana se seraient installés au sud-est de la Côte d'Ivoire, sur le littoral lagunaire (lagune ébrié) entre le XVème et XVIIème . Ils appartiennent à l'aire linguistique et culturelle $\mathrm{kwa}^{2}$. Les tchaman forment une société lignagère, organisée en génération et selon les classes d'âge. Les tchaman sont des akan. Ils partagent plusieurs éléments du groupe akan, les croyances traditionnelles, l'organisation sociale, socioculturelle et politique, l'habillement, l'art (le langage tambouriné). Cependant, les tchaman ne sont pas organisés en royaume, mais plutôt en chefferie.

"Chaque lignage constitue une unité sociale. Le chef est censé représenter la famille ou se faire représenter par autre membre de la famille lors des manifestations (...)», (DJOKE, B., 2014 :41).

Les tchaman sont matrilinéaires et l'attribution des prénoms n'est pas tributaire du jour de naissance comme chez les baoulé. Après la présentation de la culture tchaman, il convient maintenant d'aborder le cadre conceptuel, à travers les caractéristiques de la rhétorique discursive.

${ }^{1}$ «le terme tchaman (au pluriel), tchabio(masculin, singulier) et, tchabia (au féminin) avec $s a$ «tcha » est d'origine «twi », soit akan. Le suffixe « man » désigne pays en langue « twi », (DJOKE Bodjé, 2014 :35).

${ }^{2}$ L'aire linguistique kwa couvre l'est, le centre et le sud-est de la Côte d'Ivoire. Elle est composée de deux sous-groupes (les langues akan et les langues lagunaires). 


\section{La rhétorique discursive}

Le terme discours dans ce cadre, nous situe d'une part dans l'oral, précisément dans la compétence de l'oral avec ses caractéristiques. D'autre part, les contraintes de la situation de communication : une assemblée de notables qui doivent rendre un jugement à partir d'une procédure judiciaire coutumière, confère à ce discours une grammaticalité culturelle spécifique. «(..) si le texte est d'abord matière linguistique, il est aussi pratique de pensées, et objet culturel, socialement déterminé ", ( BORDAS et AL., 2015 :11). En effet, le réquisitoire et la plaidoirie sont donc mis en œuvre par les intervenants, qui cherchent à convaincre à travers un jeu d'accusation et de défense. La présence des adjectifs évaluatifs et les modalisations axiologiques, qualifient les actions en terme de causes justes, ou injustes, bien ou mal. Les modalisateurs de vérité émaillent ce discours, et permettent de former un jugement sur la vérité. Cette vérité est établie à partir des preuves techniques de la rhétorique. Les preuves peuvent être objectives, éthiques et pathétiques. Les actes de paroles mobilisés comme la narration, l'explication et l'argumentation ${ }^{3}$ nous plongent dans l'analyse du discours. « (...), le discours peut-être rapidement défini comme le résultat verbal concret de la prise de possession du matériau langagier par un sujet individuel », ou un groupe social, (BORDAS et AL., 2015 : 71). C'est un acte locutoire et illocutoire, un individu émet un énoncé avec une intention précise de provoquer des effets (l'acte devient alors selon BORDAS perlocutoire).

Par ailleurs, quelques aspects de l'ethnologie de la communication seront sollicités à travers la relation structurelle des interlocuteurs et l'emploi de formules ritualisées et proverbiales propres à la culture atchan. L' organisation des échanges verbaux respectent les caractéristiques de la rhétorique, une certaine grammaticalité culturelle.

La mise en place de l'auditoire traduit une certaine relation structurelle. En effet, la place ou le positionnement des principaux acteurs, révèlent les rôles à jouer au cours de cette interaction et le pouvoir à occuper dans les relations personnelles.

\section{La méthodologie de la recherche}

C'est une étude de type exploratoire et descriptif qui vise à faire connaître les procédures mises en place, lors d'une séance de tribunal coutumier. "La description peut constituer l'objectif même d'une recherche, par exemple dans une monographie d'un village, qui vise à faire connaître

${ }^{3}$ "L'argumentation serait indissociable de la rhétorique apparue au Ve siècle av. J-C, à l'occasion de procès destinés à restituer leurs terres aux habitants de la Sicile précédemment expatriés par une décision arbitraire. Corax composa alors le premier manuel consacré à la rédaction des plaidoiries et fonda par là même la rhétorique comme art oratoire destiné à convaincre un public », (BORDAS et AL, 2015 :138). 
tous les aspects de la réalité du village. »,(N'DA, P., 2015 :23). Décrivons ciaprès le protocole de recherche.

a) le choix du site d'enquête

Le village Akoue-djeme, avec 6500 habitants, est situé à environ 12 kilomètres d'Abidjan. Il a été choisi comme terrain de recherche, parce qu'il représente le haut lieu de la culture atchan. La facilité d'accès et la disponibilité du calendrier de la session de procédure judiciaire coutumière ont également été des critères déterminants de choix.

b) La composition de l'assistance lors du jugement coutumier

l'assistance est composée des membres du tribunal coutumier, des plaignants et de l'auditoire, (parents-es et amis-es). L'assistance est estimée à une trentaine de personnes. Le tribunal est présidé par le conseil des notables. Une dizaine de personnes constituant la notabilité du village étaient donc présents à cette audience : le chef de village, le chef des notables, le chef adjoint des notables, les porte-parole des classes d'âges, le secrétaire du conseil des notables, et des membres du conseil.

c) L'enquête

Le protocole de recueil des données s'est fait par l'observation d'une session de jugement coutumier qui s'est déroulé en mars 2021 , de $18 \mathrm{H}$ à 19H 30. Il faut signaler que, les sessions se déroulent tous les soirs de chaque vendredi ${ }^{4}$. Les faits et paroles au cours du déroulé de la séance de jugement ont été transcrits intégralement. Le type d'énoncés est donc narratif. Nous avons présenté la mise en place des principaux acteurs, le protocole ritualisé de la prise de parole et l'acte de jugement.

\section{Les résultats de l'étude}

Les résultats sont articulés autour de la mise en place, du déroulé de l'audience et de la décision de jugement.

\section{Mise en place et préambule}

D'abord et avant tout, il convient de souligner la mise en place des principaux acteurs

a) L'auditoire

Celui-ci est composé surtout de deux groupes antagonistes. Ceux-ci occupent deux rangées, deux espaces distincts. Le décors fonctionnel et significatif, permet déjà la prévention de tout débordement éventuel. C'est dire que l'occupation spatiale est un langage bien fonctionnel et un symbolisme social de grande valeur significative : c'est déjà un puissant moyen et un facteur efficace de prévention et/ou de régulation du différend. Ledit auditoire est composé, outre les parties en conflits, des amis et connaissances désireux

\footnotetext{
${ }^{4}$ Les fêtes religieuses et autres cérémonies peuvent faire modifier les jours d'audience.
} 
d'assister à la séance prochaine. L'auditoire est donc déterminé par les antagonistes et leurs relations personnelles. Nous avons également le conseil du village.

\section{b) L'arrivée du conseil du village}

Le conseil est constitué de notables, (akoube-limây), c'est-à-dire ceux qui gouvernent le village, ou les autorités administratives et politiques du village. L'arrivée de ce conseil se fait dans cet ordre.

1) D'abord les «npkoman man » ou porte-parole, précèdent le chef des notables, devançant le chef du village (akoube ote) celui qui, comme ATLAS porte le village, le garant administratif et politique qui, dès lors, clôt le cortège, la procession. Leur arrivée est suivie d'échanges de civilités.

2) Les salutations et rituels de demande de nouvelles, dans la pure tradition atchan.

Il s'agit là du « quoi de neuf», de l'objet de la séance du jour (amanien) ou nouvelle du jour. C'est au porte-parole qu'il revient ce rôle de demande de nouvelles. Pour la circonstance, la séance référencée se déroule le soir autour de 18h, il est donc question pour le porte-parole désigné (nbkômanwo), de conduire ce protocole. Il devrait tout simplement dire : «ndjuka a $a$ », en forme elliptique qui veut dire le soir, c'est-à-dire les nouvelles du soir.

Cette étape, en effet, qui constitue la $1^{\text {ère }}$ nouvelle, est adressée au plus jeune du groupe des plaignants. Il doit exposer très brièvement en quelques formules plutôt lapidaires l'objet de cette rencontre. "Il s'agit d'une salutation ou d'honorer un rendez-vous avec le chef. Il s'agit de se rapprocher de vous, du conseil pour une petite affaire, un sujet...».

Il faut noter qu' à cette première nouvelle succède une seconde :

- Généralement après la deuxième nouvelle « $\tilde{\varepsilon}$ domon $\min \mathrm{h} \varepsilon$ », il peut avoir des variantes selon les circonstances, surtout lorsque l'hôte à qui l'on demande les nouvelles a effectué un voyage, un déplacement ou a vécu un fait. L'on pourra donc dire «atchassi», « cassitech $\varepsilon$ smandjihe », ce qui signifie littéralement la route, le chemin, les nouvelles du lieu de provenance. Et la deuxième chose ? «qu'y a-t-il après les salutations annoncées? ».

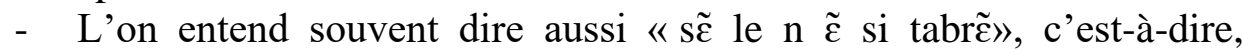
«l'homme ne marche pas d'un seul pied, il déplace l'un puis l'autre». Toute chose pour inviter à passer à la préoccupation, à l'objet réel de la visite, après ces préliminaires rituels. Car il est admis que la parole comme art se prépare sans improvisation, elle s'annonce, s'énonce avec tact, mesure par un processus, une sorte d'euphémisme traditionnel pour éviter de créer un choc, une situation désagréable, inconvenant, ménageant ainsi les susceptibilités, les émotions qui 
doivent être ainsi savamment gérées. C'est donc là une autre maîtrise de soi et la situation ambiante, qui recommande de procéder avec tact et prudence, même quand l'objet réel est connu ou semble l'être.

\section{La procédure judiciaire coutumière}

Le plaignant prend alors la parole pour formuler sa plainte, à savoir son frère aîné l'a exproprié de la parcelle de terre que leur père lui a léguée, à titre exclusif, un héritage dans sa dernière volonté. Il poursuit en affirmant : " néanmoins j'ai une immense dette de reconnaissance et de gratitude envers tous mes frères aînés, pour bienveillance et attention à mon égard depuis la plus tendre enfance jusqu'à ce jour en passant par l'éducation/formation et mon insertion socio-professionnelle. Mais pour moi tout cet élan de solidarité fraternelle ne saurait justifier un quelconque acte de dépossession de la part de ces derniers. Si c'était le cas, je demande à recevoir de ceux-ci, toute la facture des dépenses effectuées à mon sujet pour que remboursement soit fait sans délai», cf. M, R. (le plaignant).

Ả ces mots, le premier porte-parole, par un geste d'acquiescement jouant son rôle de facilitation et surtout de régulation, transmet le message reçu au deuxième, qu'il interpelle; celui-ci à son tour, le transmet au troisième, et ce dernier au chef des notables. Chaque fois par la même formule ritualisée « he mani $\varepsilon »$.

Ainsi le chef des notables demande au porte-parole, en ces termes, «nkpan manho nkpômaman», de redonner la parole au premier intervenant (le plaignant) pour l'ultime communication par ces mots « lote kroaan ». l'occasion est donnée au plaignant de donner des explications complémentaires afin d'épuiser toutes ses récriminations pour prévenir toute interférence, tout chevauchement, voire toutes réactions intempestives tendant à interrompre le prochain locuteur. L'on lui demande dans ces conditions d'examiner au besoin de compléter, de tout exposer, de fond en comble, d'être sûr que rien n'a été oublié qui lui serait préjudiciable, car il ne lui sera plus concédé un autre temps de parole, celle-ci circule, elle prend son envol...

Le plaignant prend de nouveau la parole pour ajouter ce qui suit :

«Chaque fois que l'idée m'est venue de me plaindre, à ce sujet, je n'ai reçu pour toute réponse, de la part du plus grand de mes frères aînés que des reproches relatifs à l'éducation que je devais plutôt assurer à mes nombreux enfants, de mères différentes. Je lui ai fait savoir que ce qu'il dit est vrai, juste mais ce sujet n'est pas à l'ordre du jour... » cf. M,R. (le plaignant).

Le porte-parole, pour s'assurer vraiment de la fin des propos, insiste par la formule « he min ka ? », ce qui signifie littéralement, « ta bouche s'arrête là ? », ou encore « est-ce là le point de chute de ta bouche? ». 
La réponse est affirmative, alors suit la procédure de transition par la

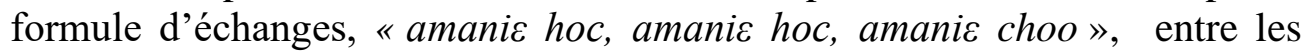

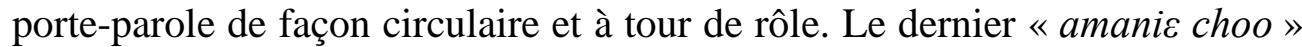
est à l'intention du chef des notables.

La parole revient alors au frère-aîné, le prétendu expropriateur qui adresse ses remerciements à la notabilité, signe de civilités avant tout propos ; «je souhaite m'excuser, la parole est difficile », "abrveto coman, ale li le pan kubré agnin brveto apâsâ romĩ a pa sa he mĩ », « tout le monde n'est pas à l'aise à manipuler la parole en toutes circonstances, surtout devant un public quel qu'il soit ». Telle précaution oratoire n'est pas sans intérêt, de la part du frère aîné. Ce, pour extirper d'éventuelles arêtes, d'éventuels écarts langagiers, sur le plan formel notamment.

Puis celui-ci en vient au vif du sujet :

«permettez-moi maintenant, honorables notables, honorables membres du conseil pour l'occasion que vous me donnez de faire la lumière sur ce problème foncier familial crée de toutes pièces. Les propos du frère cadet sont une série d'erreurs et de contre-vérités. En voici quelques éléments de preuve. De nous deux qui est l'aîné? Pourquoi c'est à lui que notre défunt père confie à titre exclusif la propriété du terrain, objet du litige de ce jour? A-t-il assisté papa? Était -il présent aux derniers jours, aux dernières heures de notre défunt père pour parler de la dernière volonté de celui-ci, en termes d'héritage foncier? Qu'il nous donne la preuve de sa présence! Pour quelles raisons lui et non un autre des frères pour jouir du droit exclusif de cet héritage (adjahi), di ti ké un ni lo, c'est quand la biche s'est entourée de vertus telles la vigilance, la prudence, la patience...qu'elle devient âgée, qu' elle s'assure un âge d'heureuse vieillesse », cf. M, J. (l'accusé).

Le frère aîné, en utilisant cet énoncé proverbial, veut prodiguer des conseils à son cadet, mais vise surtout l'assemblée ; le proverbe pour susciter l'adhésion autour d'une vérité générale et philosophique.

"Dans l'Afrique, le proverbe est utilisé pendant les séances de tribunaux populaires sous l'arbre à palabres, précisément entre les hommes. Ces séances sont de véritables joutes oratoires où l'on allie le verbe à la maîtrise orale. », (TOLA,T., $2017: 27)$.

"Ha nin ni wawwa nan», "que le frère cadet n'ait pas la préoccupation, l'obstination de celui qui cherche à se désaltérer avec un extrême empressement, une hâte jamais égalée. Il faut de la patience, de la mesure et de la modération. Des investigateurs tapis dans l'ombre de la grande famille tirent les ficelles, spécialistes des conflits et des différends, ils le sont. Ceux-ci sont découverts à leur 
insu. Pourquoi de telle déclaration mensongère du frère cadet? Exposant en public, un fait strictement familial. Se soucie-t-il un instant de la mise en valeur du terrain paternel, comme tous les autres frères et sœurs. La preuve! Je l'ai convié le $1^{\text {er }}$ janvier 2014, en lui présentant les voux traditionnels au téléphone, comme d'habitude, à joindre aux autres frères pour l'exploitation de la parcelle, en l'occurrence bâtir une maison familiale commune. Je lui ai même demandé une contribution financière de trois millions de francs CFA. Les témoins de ce coup de fil, de cette conversation téléphonique sont encore vivants, par la grâce de Dieu. Mon frère a répondu par des éclats de rires, puis a relancé par ces mots : "combien de francs?". J'ai repris et insisté trois millions! Depuis lors, mon frère a cessé tout contact avec moi. Plus de coup de fil, plus d'échanges fraternels. Pire, il m'évite, ne donne aucune suite à mes appels...Et, voilà qu'aujourd'hui, il me traduit devant le conseil des notables. Je dois ajouter que devant son silence, j'ai entrepris les travaux qui sont au stade de la finition, sinon à la toiture, pour être précis» cf. M,J. (l'accusé).

Comme précédemment, les porte-parole ont demandé, «di mwa li pôh », «lo kroa a a», un temps de réflexion; si d'aventure, il y avait des ajouts de compléments d'informations à prendre en compte pour évaluer la situation du différend familial.

L'aîné a tout simplement ajouté ceci : "l'on ne peut être aussi ingrat comme le démontre mon jeune frère, si ce n'est mû par des mobiles inavoués ».

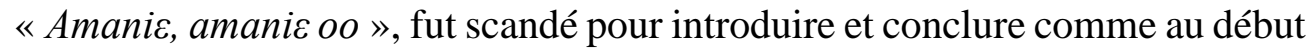
de la relance de la procédure.

Les porte-parole ont juste voulu poser des questions d'éclaircissement au frère cadet. Celles-ci sont résumés en ces points :

- Le cadet reconnaît-il avoir été sollicité pour la mise en valeur de la parcelle référencée?

- La somme avancée de trois millions peuvent-ils couvrir une dépense de construction?

- Ne faudrait-il pas plutôt voir en cela une volonté de recherche de l'unité familiale?

- N'est-ce pas là pour le frère aîné un appel à une unité d'action dans la convivialité pour maintenir la cohésion familiale?

Ả l'ensemble de ces questions d'éclaircissement, le cadet a eu comme toute réponse, "je veux mon terrain et rien d'autre. Je n'ai nullement l'intention de perdre cet héritage foncier. », a-t-il assené. 
Reprenant la parole, le premier intervenant parmi les n'kpômanman (porte-parole) invite ses pairs à poursuivre en disant : «abweto kale, lopan enin bweto han ge tว», "le temps de la parole est arrivé, celui qui désire s'exprimer sur le sujet le peut maintenant».

La phase de délibération se fera selon le droit coutumier foncier atchan. "Toute société, si peu évoluée soit-elle, se donne des règles et des institutions qui se complètent et s'enrichissent au fur et à mesure que progresse son organisation et que les rapports entre les individus qui la composent deviennent plus complexes », (TOLA, T., 2017 : 71).

\section{La délibération ou la décision du tribunal coutumier}

Cette phase est d'une grande importance. Il laisse présager, sauf erreur ou omission, la décision finale. Ainsi l'un après l'autre $n$ 'kpômanman ont des propos tout à fait convergents, guidés par la sagesse atchan, qui prône l'apaisement pour une cohésion familiale, gage d'une paix véritable entre membres d'une famille issue de la communauté villageoise.

En substance et dans une vision de synthèse, il n'est pas question de démolir les bases d'une construction largement entamée pour faire plaisir au jeune frère, qui se dit "héritier», désigné par le défunt père. Ce serait un fâcheux précédent, voire une grave menace pour la gestion du pouvoir dans le village, pour la société atchan.

\section{Vers une conclusion du tribunal coutumier}

Au moment de conclure, le chef des notables eut cette recommandation qui a fait l'unanimité, à savoir, "je demande, dit-il à l'aîné des aînés, au plus âgé des frères une ultime concertation familiale restreinte, sur la base de ce qui vient d'être dit, de procéder à un enregistrement des parcelles familiales. Il y aura bien un terrain non bâti pour le céder au frère cadet plaignant en compensation de la parcelle, objet du litige, pourvu que ce terrain soit dans le périmètre foncier du village d'Akoué-djemé, sous l'autorisation administrative de notre chef bien-aimé », cf. A, A. (le chef des notables).

Ces mots sont d'un trait de génie, une inspiration des ancêtres, s'exclama le public. Et le doyen du lignage d'affirmer, dans un soulagement, "lo bwe we nin we $\eta e$ ». C'est la parole attendue, c'est la parole que nous voulons entendre, " $a$ bwe paya», a renchéri le porte-parole en chef «nkpomanho » par cette formule, «abwe paya»; mot à mot «la parole est dans les cimes des arbres », ce qui veut dire que tout est dit et bien dit, voire conclu définitivement ou plus rien ne doit être ajouté.

Avant la levée de la séance concernant le présent litige, le doyen du lignage a remercié, par un vibrant hommage la notabilité qui peut poursuivre sa séance hebdomadaire. Non sans avoir adressé les bras levés aux cieux un 
merci immense aux ancêtres tutélaires, surtout à Dieu source d'amour et de tout bienfait, «yâkan nansin, adominiga nansin ».

Les phénomènes sociaux sont complexes, ils ne peuvent pas se résumer en une simple description, sans une explication de tout le processus interactionnel et environnemental. Quelles sont les limites de l'étude ?

\section{Discussion}

Cette recherche descriptive a voulu mettre en relief la rhétorique discursive lors d'une séance de tribunal coutumier. Les énoncés narratifs peuvent par moments, donner à cette étude une allure anecdotique. Cependant, les discours rapportés dans ce cadre sont des objets sociolinguistiques, qui s'inscrivent dans l'analyse de discours.

«On ne parle plus aujourd'hui d'analyse du discours, mais d'analyses de discours ; cette approche plurielle est bien illustrée (...) par la diversité des genres de textes abordés en relation avec le contexte de leur production: conversations, entretiens, débats, consultations, interrogatoires (...). » (CUQ, J-P., 2003 :74).

La transcription simple de cette séance de jugement, a pu limiter notre compréhension des éléments observés. Toutefois, cette approche, nous a permis de ne pas influer sur le cours des événements.

$\mathrm{Du}$ point de vue du règlement des conflits par les instances coutumières, nous retrouvons les caractéristiques communes des mécanismes ${ }^{5}$ traditionnels ouest-africains mentionnés par BAGAYOKO, $\mathrm{N}$. et FAHIRAMAN,K. (2017). L'importance de la parole ritualisée, en effet, donne une dimension sacrée à un fait sociologique, comme la gestion d'un conflit. La nécessité de cohésion sociale guide en général, les décisions de jugement. Le spirituel est sollicité pour y parvenir. Dans le cadre traditionnel de gestion des conflits, d'autres mécanismes de régulations sociales, comme les alliances à plaisanterie ou les rituels ordaliques ${ }^{6}$ sont également pratiqués en Côte d'Ivoire et en Afrique de l'ouest.

«Globalement les parentés et les alliances à plaisanteries sont des institutions sociales dont l'objectif est de garantir durablement la paix et l'harmonie au sein des familles, entre clans, entre groupes

\footnotetext{
${ }^{5}$ L'oralité, la dimension rituelle, la référence à l'histoire, le souci primordial de sauvegarder la cohésion sociale, la sacralité et l'imbrication du spirituel et du temporel, l'importance de la dimension spatio-temporelle, sont les caractéristiques communes des mécanismes traditionnels ouest-africains de gestion des conflits (BAGAYOKO, N. et FAHIRAMAN,K. (2017).

${ }^{6}$ Les rituels ordaliques sont pratiqués pour rechercher la vérité et apporter une paix définitive. Il s'agit pour le belligérant de boire un breuvage qui n'épargne que les innocents.
} 
socioprofessionnels, entre castes et entre ethnies. »,(BAGAYOKO, N. et FAHIRAMAN,K., $2017: 29$ ).

Il faut signaler qu'en général, les alliances à plaisanteries se déroulent dans un cadre convivial et concernent des personnes de groupes claniques et ethniques différents. Toutefois, ces mécanismes sont sollicités lors des règlements de conflits, pour éviter tout débordement. Car ces alliances reposent sur les principes de respect de la vie humaine, et d'obligation d'assistance et de fraternité. Il y a d'autres mécanismes de régulation sociale, comme la danse « adjanou ${ }^{7}$ » chez les akan ou l'association « usana ${ }^{8}$ » au Sénégal, qui se traduisent par des actions de femmes, qui peuvent investir les lieux publics pour exiger la paix. Ces actions sont des prières, des paroles et chants pour purifier la terre qui a été souillée par de mauvais agissements. Cependant, ces dispositifs traditionnels de règlement des conflits, ne suffisent pas pour gérer toutes les complexités de situations belligérantes générées, par la modernité et la prise en compte des exigences internationales.

\section{Conclusion}

La séance oratoire s'articule bien autour de trois grandes parties, à savoir :

- la présentation ou l'introduction,

- la production du message ou le développement,

- le verdict ou la conclusion.

Ce démembrement renforce l'idée d'une parole organisée, structurée. Celle-ci n'est pas, comme l'on a une tendance à le croire, « un palabre », dans la conception plutôt négative et dévalorisante de la notion. Le modèle conversationnel obéit aux principes ${ }^{9}$ de régulation des échanges.

En outre, il se manifeste au sein de ce temps de parole un jeu intertextuel savamment orchestré, en l'occurrence à travers des formules proverbiales (ou dictons). Une ingénierie didactique, qui en fonction de l'objectif fixé transmet les enseignements souhaités. Le modèle

${ }^{7}$ L'adjanou est une danse akan, pratiquée par des femmes nues qui sortent en public lors de graves crises de la société.

${ }^{8}$ Les usana sont des associations de femmes «crées par des femmes âgées aux pouvoirs «mystiques » reconnus et autour desquelles se forme un chapitre de suivantes, interviennent en cas de malheur privé ou public en organisant des sacrifices et des cérémonies de prières. », (FOURCHER, V., 2007)

${ }^{9}$ GRICE Paul.H formule quatre règles de coopération au cours des échanges :

- Maxime de quantité : « donner toutes les informations nécessaires ».

- Maxime de qualité : « ne tenir que des propos vrais et prouvés ».

- Maxime de relation : «parler à propos ».

- Maxime de modalité : «s'exprime clairement et sans ambiguïté », (BORDAS et AL., 2017 :146). 
conversationnel des règlements des conflits fonciers en pays atchan est révélateur de la compréhension que les tchaman ont du droit coutumier. Ce droit est également la vision d'un monde régulé par le respect des ancêtres. Le récit de cette audience montre l'importance de la prise de paroles. La disposition des participants en amont, est une véritable scénographie qui prévient les débordements tout en invitant à l'expression de la pensée.

\section{References:}

1. BAGAYOKO, N. et FAHIRAMAN, K., (2017). Les mécanismes traditionnels de gestion des conflits en Afrique subsaharienne. Chaire RAOUL-DANDURAND. UQAM. Montréal.

2. BORDAS, E., BAREL-MOISAN, C., BONNET,G., DÉRUELLE,A.,

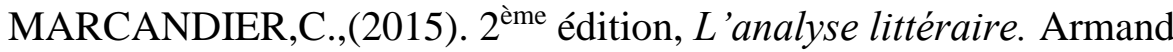
Colin. Paris.

3. BÔLE-RICHARD. R. (2017). Nouvelle édition. Introduction à l'écriture et à l'orthographe de l'ébrié. EDILIS. Abidjan.

4. CUQ, J-P.,(2003). Dictionnaire de didactique du français, langue étrangère et seconde. CLE International. Paris.

5. DJOKE,B., (2014). Musique dans la société atchan. Côte d'Ivoire.

6. FOURCHER, V., (2007). " Tradition africaine et résolution des conflits. Un exemple sénégalais », dans Politix 2007/4 (n80). PP5980.

7. HAMPATÉ BÂ, A., (1972). Aspects de la civilisation africaine. Présence africaine.

8. N'DA, P., (2015). Recherche et méthodologie en sciences sociales et humaines. L'Harmattan. Paris

9. TOLA,T., (2017). Épopée: une synergie des genres oraux, des sciences humaines.Éditions Universitaires Européennes.

10. ZADI Zaourou, B., (2011). Anthologie de la littérature orale de Côte d'Ivoire. Harmattan. Burkina. 